\title{
The value of adding sub-valvular procedures for chronic ischemic mitral regurgitation surgery: a meta-analysis
}

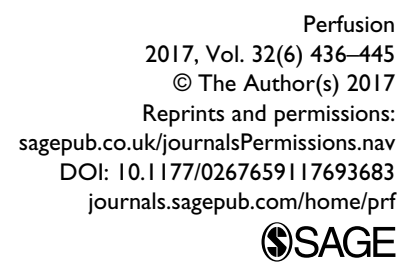

(S)AGE

\author{
Marco Moscarelli, ${ }^{1,2}$ Thanos Athanasiou, ${ }^{3}$ Giuseppe Speziale, ${ }^{2}$ \\ Prakash P. Punjabi, ${ }^{3}$ George Malietzis, ${ }^{3}$ Patrizio Lancellotti ${ }^{4}$ \\ and Khalil Fattouch ${ }^{5}$
}

\begin{abstract}
Background: The most performed repair technique for the treatment of chronic ischemic mitral regurgitation in patients referred for bypass grafting remains restricted annuloplasty. However, it is associated with a high rate of failure, especially if severe tenting exists.

Objectives: To understand if adjunctive sub-valvular mitral procedures may provide better repair performance.

Methods: A systematic literature review identified six studies of which five fulfilled the criteria for meta-analysis. Outcomes for a total of 404 patients (214 had adjunctive sub-valvular procedures and 190 restricted annuloplasty) were meta-analyzed using random effects modeling. Heterogeneity and subgroup sensitivity analysis were assessed. Primary endpoints were: late recurrence of moderate mitral regurgitation, left ventricle remodeling and coaptation depth at follow-up. Secondary endpoints were: early mortality, mid-term survival and operative outcomes.

Results: Sub-valvular procedure technique was associated with a significantly lower late recurrence of mitral regurgitation (Odds ratio (OR) $0.34,95 \%$ Confidence Interval $(\mathrm{Cl})[0.18,0.65], \mathrm{p}=0.0009)$, smaller left ventricle end-systolic diameter (Weighted Mean Difference (WMD) $-4.06,95 \% \mathrm{Cl}[-6.10,-2.03], \mathrm{p}=0.000 \mathrm{I}$ ) and reduced coaptation depth (WMD $-2.36,95 \% \mathrm{Cl}[-5.0 \mathrm{I},-0.7 \mathrm{I}], \mathrm{p}=0.009)$. These findings were consistent, even in studies that included patients at high risk for repair failure (coaptation depth $>10 \mathrm{~mm}$ and tenting area $>2.5 \mathrm{~cm}^{2}$ ). A low degree of heterogeneity was observed. There was no difference in terms of early mortality and mid-term survival; sub-valvular technique was associated with prolonged cardiopulmonary and cross-clamp time.

Conclusions: Adding sub-valvular procedures when repairing ischemic chronic mitral valve regurgitation may be associated with better durability, even in the case of the presence of predictors for late failure.

Perspective: Surgical sub-valvular adjunctive procedures have to be considered in the case of the presence of echocardiographic predictors for late failure.
\end{abstract}

\section{Keywords}

mitral regurgitation; mitral valve repair; ischemic mitral valve; statistics; meta-analysis

\section{Introduction}

The most performed surgical repair procedure for the treatment of chronic ischemic mitral regurgitation (cIMR) is the restrictive annuloplasty alone (RA). ${ }^{1}$ However, mechanisms that cause mitral regurgitation (MR) are numerous, such as left ventricle remodeling, displacement of papillary muscles and tethering of the valve with loss of leaflet coaptation. ${ }^{2}$ Addressing mitral annulus dilatation alone may not guarantee a durable correction. Also, the left ventricle may be subject to continuous remodeling, hence, replacement may be preferred, particularly in the case of severe cIMR. ${ }^{3}$ Many authors
'NHLI, Imperial College London, London, UK

${ }^{2}$ GVM Care and Research, Anthea Hospital, Bari, Italy

${ }^{3}$ Department of Surgery and Cancer, Imperial College London, London, UK ${ }^{4}$ Department of Cardiology, GIGA Cardiovascular Sciences, University of Liège Hospital, Liège, Belgium and GVM Care and Research Group, Maria Eleonora, Palermo, Italy

${ }^{5}$ GVM Care and Research, Maria Eleonora, Palermo, Italy

Corresponding author:

Marco Moscarelli GVM Care and Research, Anthea Hospital, Via

Camillo Rosalba 35/37 Bari, Italy.

Email: m.moscarelli@imperial.ac.uk 


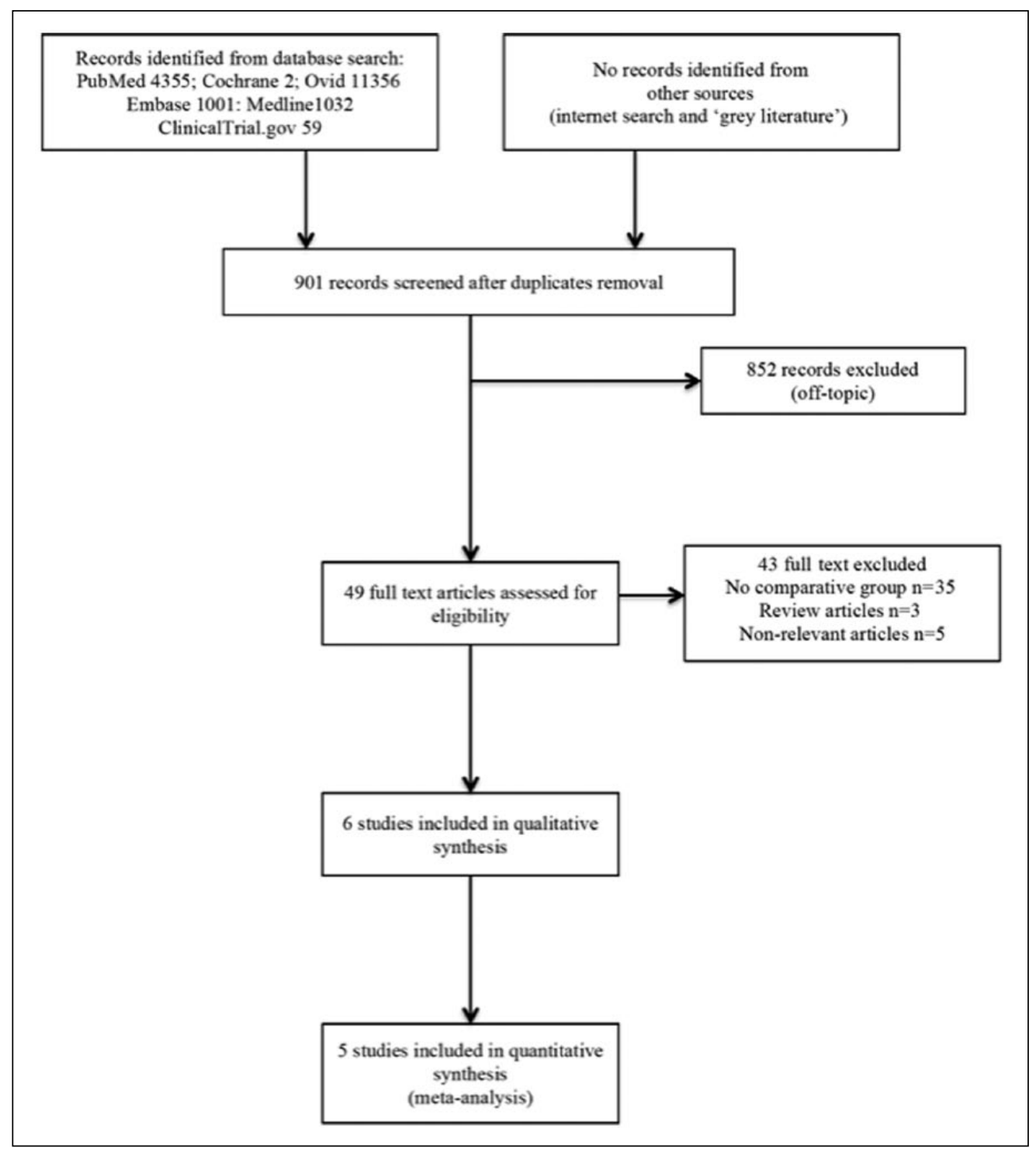

Figure I. Search Strategy.

have highlighted benefits in terms of repair durability by adding sub-valvular procedures (SVP) such as papillary muscle relocation (PMR), elevation (PME) or approximation (PMA) and chordal cutting (CC). ${ }^{4}$ However, concerns exist in terms of safety since such techniques may increase the operating time. The main aims of this study were to identify whether: 1) SVP may offer reduced recurrence of late cIMR (defined as per MR $\geqslant 2+$ ) when compared with annuloplasty alone; 2 ) if it is associated with better left ventricle geometry; and 3) if there are any increased operative risk.

\section{Material and Methods}

\section{Literature search}

The search was performed using PubMed, Ovid, Embase, Medline and Cochrane databases, using
$\mathrm{MeSH}$ terms 'ischemic mitral regurgitation', 'restrictive annuloplasty', 'papillary muscle relocation / approximation / elevation', 'chordal cutting'. In addition, our search was extended to include the clinicaltrials.gov database and 'grey' literature for further rigor. The 'related articles' function in PubMed was also used to ensure completeness. The last date for this search was $1^{\text {st }}$ September 2015 (Figure 1).

\section{Inclusion and exclusion criteria}

All articles reporting outcomes for SVP (experimental group) and RA (control group) were included. Studies were excluded from the review if: (1) Inconsistency of data did not allow valid extraction; (2) duplicated data; (3) if the experimental or control group was robotic intervention and (4) the trial/study was carried out on 
animal models. Based on these criteria, two assessors (MM, SC) independently selected studies for further examination by title and abstract review. All potentially eligible studies were retrieved in full for further evaluation. Any disagreement was resolved by discussion with a third author (KF, TA). Statistical concordance testing was performed using Cohen's Kappa coefficient to measure of inter-rate agreement.

\section{Data analysis}

Two authors (MM, SC) independently extracted the following data from each paper using a predefined protocol, including: first author; year of publication; study type; number of subjects and study population demographics. Specific outcome data was, where possible, for the following: (i) Primary endpoints: late recurrence of cIMR, LVEDD (left ventricular end diastolic diameter), LVESD (left ventricular end systolic diameter), CD (coaptation depth), LVEF (left ventricular ejection fraction), (ii) Secondary endpoints, including: cardiopulmonary bypass time (CPB), cross-clamp time (XCT) early mortality (defined as 30 days or in-hospital mortality) and survival. Meta-analysis was performed in line with recommendations from the Cochrane Collaboration and in accordance with both PRISMA (Preferred Reporting Items for Systematic Reviews and Meta-Analyses) and MOOSE (Meta-analysis OfObservational Studiesin Epidemiology) guidelines. ${ }^{5,6}$ The analysis was conducted by use of Review Manager $^{\circledR}$ Version 5.1.7 for Windows (The Cochrane Collaboration, Software Update, Oxford, UK) and STATA v.11 statistical analysis software. Data were analyzed using a weighted DerSimonian-Laird random effects model. Continuous data were investigated using weighted mean difference (WMD) as the summary statistic, reported with $95 \%$ confidence intervals (CI). The point estimate of the WMD was considered statistically significant at $\mathrm{p}<0.05$ if the $95 \%$ confidence interval did not include the value zero. Categorical variables were analyzed using the odds ratio (OR). An OR of $<1$ favored the treatment group and the point estimate of the OR is considered statistically significant at the $\mathrm{p}<0.05$ level if the $95 \%$ confidence interval does not include the value 1.

\section{Heterogeneity}

Inter-study heterogeneity was explored using the $\mathrm{Chi}^{2}$ statistic, but the $\mathrm{I}^{2}$ value was calculated to quantify the degree of heterogeneity across studies that could not be attributable to chance alone. When $\mathrm{I}^{2}$ was more than $50 \%$, significant statistical heterogeneity was considered to be present. Two strategies were used to assess data validity and heterogeneity: (1) analysis of higher quality studies (quality score $>13$ ) and sensitivity studies that included patients at high risk for recurrence of MR; (2) Funnel plots to evaluate publication bias.

\section{Quality scoring}

Quality assessment of each study was performed by attributing a quality assessment score using a modification of the Newcastle-Ottawa scale ${ }^{6}$ that included preoperative echocardiographic findings, clinical baseline characteristics and operative procedures. Studies attaining greater than the median score of 13 (out of a maximum 27) were defined to have 'higher quality matching'. Modified Newcastle-Ottawa scoring criteria are shown in Table 1.

Studies that included patients at a high risk for failure and late recurrence of cIMR, such as CD $>10 \mathrm{~mm}$ and TA $>2.5 \mathrm{~cm}^{2}$, were part of the sensitivity analysis.

\section{Risk of bias analysis}

A domain-based evaluation of risk of bias was performed in accordance with the guidelines outlined in the Cochrane Handbook for Systematic Reviews of Interventions Version 5.1.0.7 Two authors (MM, SC) subjectively examined all the studies included in this review and assigned a value of 'yes', 'no' or 'unclear' to the following questions: (i) Was the allocation sequence adequately generated? (ii) Was the allocation adequately concealed? (iii) Was there blinding of participants, personnel and outcome assessors? (iv) Were incomplete outcome data sufficiently assessed? (v) Are reports in the study free of the suggestion of selective outcome reporting? 'Risk of bias' plots were performed using Review Manager Version 5.1.7 for Windows.

\section{Results}

Our search revealed five studies ${ }^{8-12}$ fulfilling these inclusion criteria, producing a pooled data set of 1254 patients of whom 228 underwent SVP and 203 underwent RA (Table 2). There was 100\% concordance between reviewers equating to a Cohen's kappa coefficient of $\kappa=1$.

Two studies were propensity matched, ${ }^{9,10}$ two were retrospective observational ${ }^{8,12}$ and one case control. ${ }^{11}$ One study ${ }^{13}$ did not provide follow-up, hence, it was not included in the quantitative synthesis.

\section{Surgical definitions and operative characteristics}

Sub-valvular procedures were defined as adjunctive surgical procedures at the level either of the papillary muscles or the chordae. In order not to increase surgical heterogeneity, posterior leaflets techniques were not included in the analysis. Wakasa ${ }^{8}$ achieved papillary muscle approximation (PMA) using the trans-valvular or trans-ventricle approach; the latter was used in the 
Table I. Criteria for quality assessment.

Quality checklist

Selection

(I) Assignment for treatment - any criteria reported? (If yes, I star)

(2) How representative was the reference group (RA) in comparison to the general population for mitral surgery? (If yes, I star; no star if the patients were selected or selection of group was not described)

(3) How representative was the reference group (SVP) in comparison to the general population for mitral surgery? (If drawn from the same community as the reference group, I star; no star if drawn from a different source or selection of group was not described)

Comparability based on echocardiographic characteristics (I star each)

(4) Grade of cIMR; (5) mean tenting area; (6) mean coaptation depth; LVEDD mm; (7) LVESD mm; (8) mean LV ejection function. Comparability based on pre-operative characteristics (I star each)

(8) age; (9) gender; (I0) renal function; (II) extracardiac arteriopathy; (I2) poor mobility; (13) previous cardiac surgery; (14) chronic lung disease; (I5) active endocarditis; (16) critical pre-operative state; (I7) IDDM; (I8) NYHA; (19) CCS IV; (20) recent MI; (2I) pulmonary hypertension; (22) urgency; (23) combined.

Comparability based on number of bypass grafts performed or ring size (I star each)

(24) Number of distal anastomoses per patient

(25) Ring size

Outcome assessment

(26) Clearly defined outcome of interest (If yes, I star).

(27) Echocardiographic and clinical follow-up (I star if described).

CABG: coronary artery bypass grafting; CCS: Canadian class society; IDDM: insulin dependent diabetes mellitus; cIMR: chronic ischemic mitral regurgitation; LVEDD: left ventricular end diastolic diameter; LVESD= left ventricular end systolic diameter; MI: myocardial infarction; NYHA: New York Heart Association; RA: restrictive annuloplasty; SVP: sub-valvular procedure.

case of large left ventricle volume (LVEDD $\geqslant 65 \mathrm{~mm})$ with ischemic scar. ${ }^{8}$ Papillary muscle relocation (PMR) was used in the Fattouch ${ }^{10}$ and Langer ${ }^{11}$ series (RING + STRING). Calafiore ${ }^{9}$ carried out chordal cutting (CC) of all second-order chordae while Borger ${ }^{12}$ performed CC arising specifically from the infarcted papillary muscles. A moderate to true undersizing technique was used in the ring alone group, ${ }^{8}$ whereas, in the SVP group, a truesized ring was generally preferred (Table 2 ). A modified Newcastle-Ottawa scale was used to check pre-operative comparability (Table 1).

\section{Primary outcome}

A summary of both primary and secondary endpoints is shown in Table 3. At a mean follow-up of 35.2 months, the SVP group was associated with a statistically significant reduced recurrence of cIMR $\geqslant 2+(\mathrm{OR}$ $0.33,95 \%$ CI $[0.16,0.66], \mathrm{p}=0.002)$ with no heterogeneity $\left(\mathrm{Chi}^{2} 4.43, \mathrm{I}^{2} 10 \%, \mathrm{p}=0.35\right)$, smaller LVESD (WMD -4.06, 95\% CI $[-6.10,-2.03], \mathrm{p}=0.0001$ ) with no heterogeneity $\left(\mathrm{Chi}^{2} 0.30, \mathrm{I}^{2} 0 \%, \mathrm{p}=0.86\right)$ and reduced CD (WMD -2.36, 95\% CI [-4.28, -0.45], $\mathrm{p}=0.02$ ) with heterogeneity $\left(\mathrm{Chi}^{2} 206.98, \mathrm{I}^{2} 99 \%, \mathrm{p}=0.00001\right)$. Left ventricular end diastolic diameter did not differ at follow-up (WMD -0.72, 95\% CI [7.77, 6.32], $\mathrm{p}=0.84$ ) as with the LVEF (WMD -1.39, 95\% CI [-7.48, 4.71], $\mathrm{p}=0.66)$. However, high heterogeneity was observed for both LVEDD ( $\mathrm{p}=0.00001)$ and $\operatorname{LVEF}(\mathrm{p}=0.00001)$ (Figures $2 \mathrm{a}-\mathrm{b} /$ Figures 3a-b / Figure 4).

\section{Secondary endpoints}

In the SVP group, a prolonged CPB time was observed (WMD 9.73, 95\% CI [2.37, 17.09], $\mathrm{p}=0.01$ ) together with prolonged XCT (WMD 7.52, 95\% CI 2.96, 12.07, $\mathrm{p}=0.001)$. There was no difference in terms of early mortality (in-hospital mortality / 30 days mortality) (OR $0.92,95 \%$ CI $[0.42,2.03], \mathrm{p}=0.84$ ) and of mid-term survival (hazard ratio (HR) $0.79,95 \%$ CI $[0.45,1.40]$, $\mathrm{p}=0.42)$. Heterogeneity was observed for XCT $(\mathrm{p}=0.004)$, but not for CPB, early mortality and survival (all causes mortality) ( $\mathrm{p}=0.23,0.83,0.38$, respectively) (Table 3 ).

\section{Quality scoring and sensitivity analysis}

An implemented version of the Newcastle-Ottawa scale was built. Comparability was calculated, assigning 1 point (star) for each pre-operative echocardiographic characteristic (Grade of cIMR, mean tenting area, mean coaptation depth, LVEDD, LVESD; LVEF), for each EuroSCORE II risk factors and for operative characteristic such as number of distal anastomosis and ring size. Criteria of selection, outcomes assessment and follow-up were also included. The overall quality of the studies is outlined in Table 4. All of the studies included in this review were considered to be of high quality, scoring above the median of 13 (of 27 points) (Table 2). Three studies that included patients with specific predictors of failure and recurrence of cIMR, such as CD $\geqslant 10 \mathrm{~mm}$ and / or TA $\geqslant 2.5 \mathrm{~cm}^{2}$ were included in a sensitivity analysis 


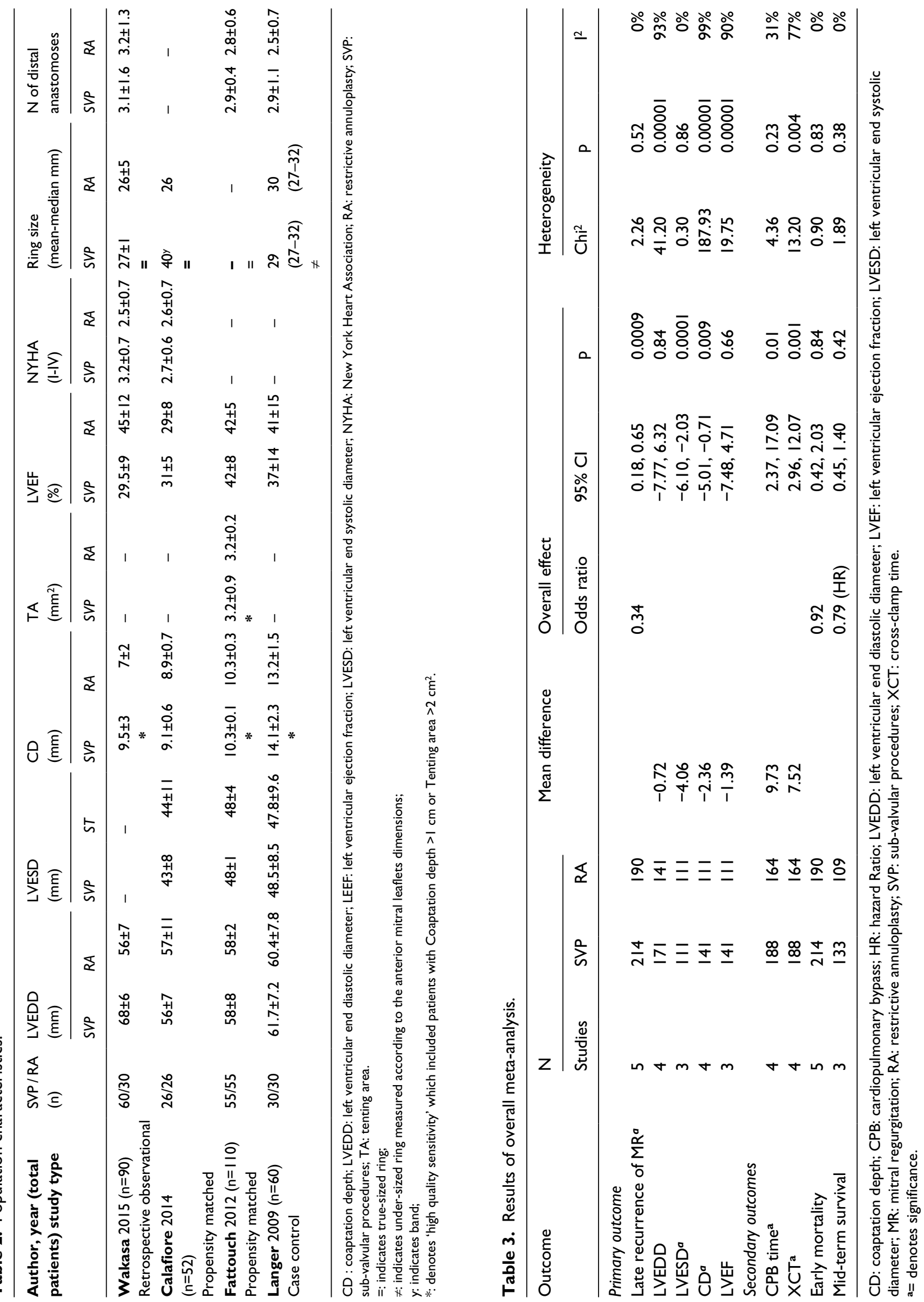


(a)
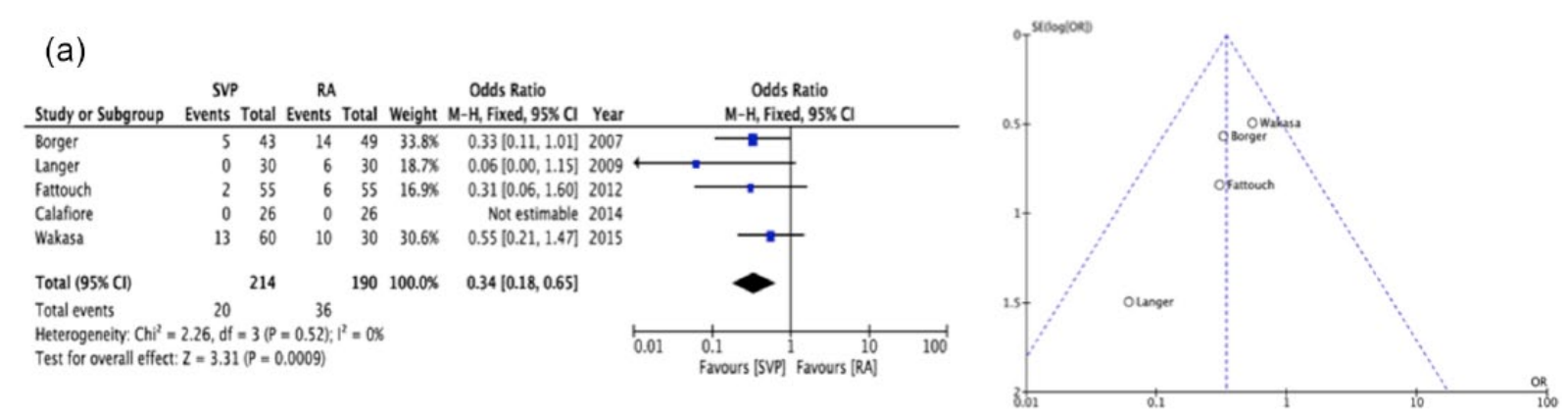

(b)
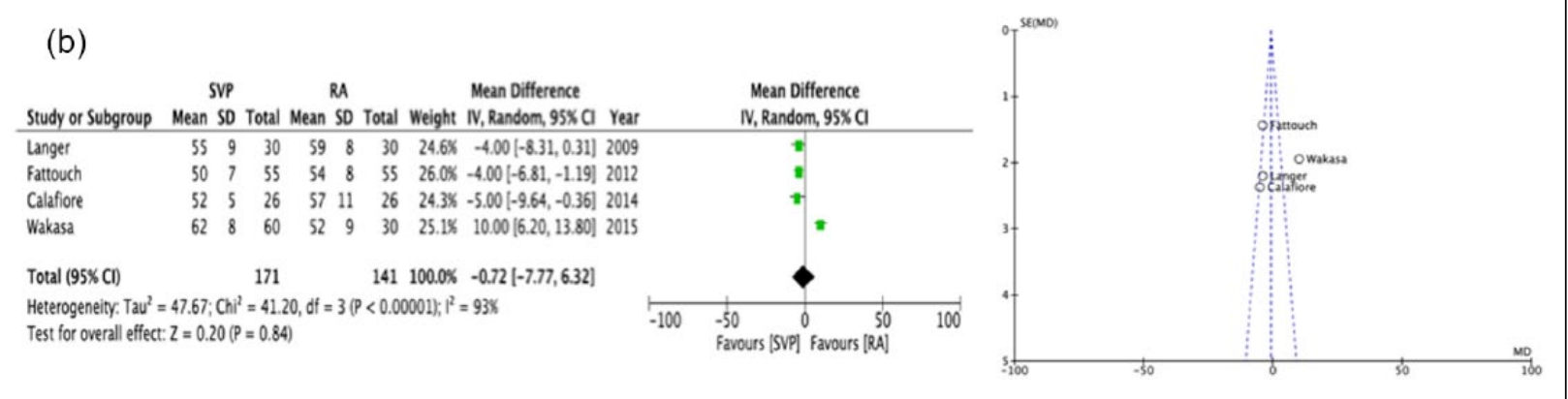

Figure 2. Forest and Funnel plots [SVP] vs [RA]: (a) late recurrence cIMR (b) LVEDD.

(a)
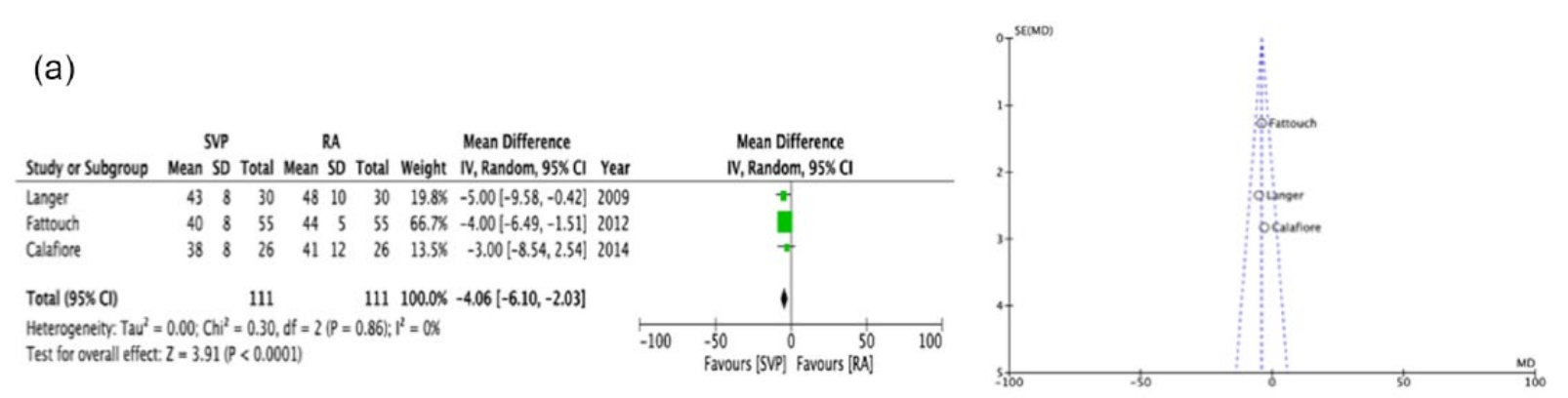

(b)
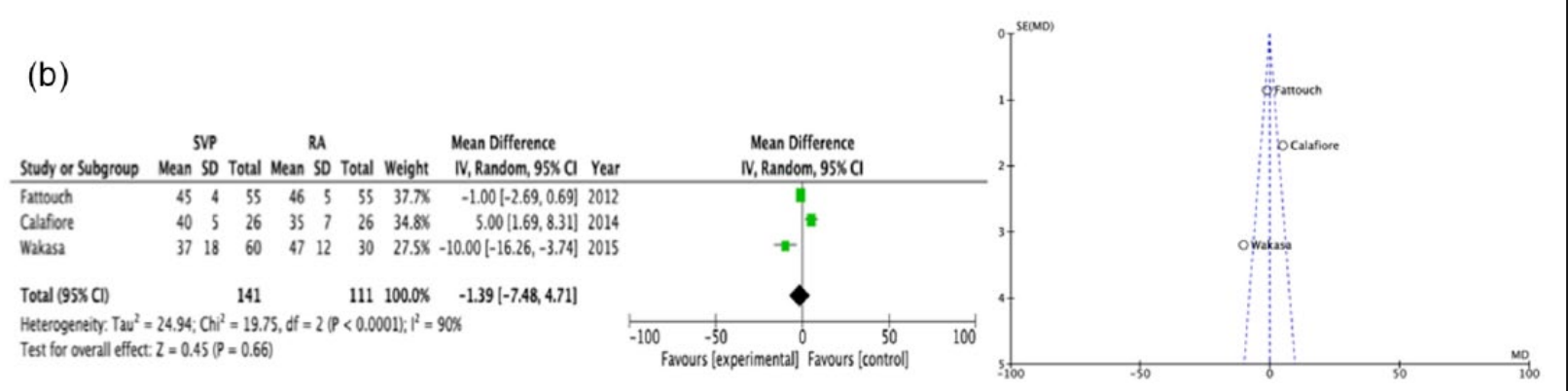

Figure 3. Forest and Funnel plots [SVP] vs [RA]: (a) LVESDD (b) CD.

(Table 5). A statistically significant lower recurrence of cIMR was observed in this SVP group (OR 0.39, 95\% CI $[0.16,0.95], \mathrm{p}=0.04)$ with no heterogeneity $(\mathrm{p}=0.33)$ as well as smaller LVESD (WMD -4.23 , 95\% CI [-6.42, -2.04], $\mathrm{p}=0.0002)$ with no heterogeneity $(\mathrm{p}=0.71)$ and reduced CD (WMD -3.99, 95\% CI [-4.15, -3.82], $p=0.0001)$ with no heterogeneity $(p=0.31)$. In terms of secondary outcomes, while XCT was statistically significantly prolonged in the SVP group ( $\mathrm{p}=0.004)$, no significant different was observed for the CPB time ( $\mathrm{p}=0.07)$; there was no difference with regard to early mortality or mid-term survival ( $\mathrm{p}=0.88$ and 0.56 , respectively). 


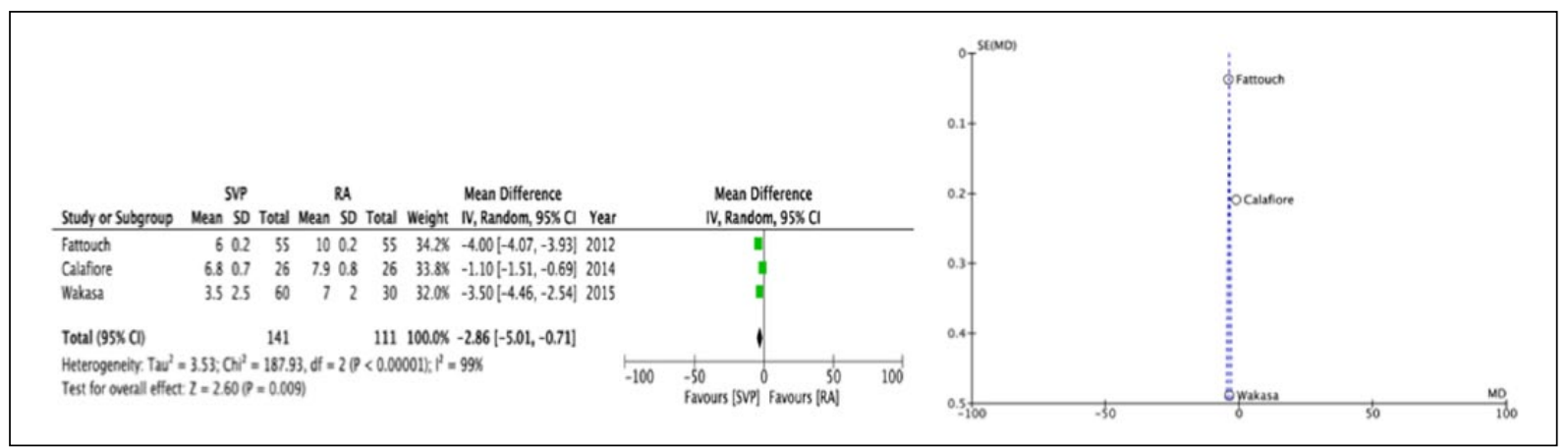

Figure 4. Forest and Funnel plots [SVP] vs [RA]: LVEF.

Table 4. Quality scoring.

\begin{tabular}{|c|c|c|c|c|c|c|c|c|c|}
\hline \multirow[t]{2}{*}{ Authors ( $\mathrm{N}$ of patients) } & \multicolumn{3}{|c|}{ Selection } & \multicolumn{3}{|c|}{ Comparability } & \multicolumn{2}{|c|}{ Outcome } & \multirow[t]{2}{*}{ Total } \\
\hline & I & 2 & 3 & 4 & 5 & 6 & 7 & 8 & \\
\hline Wakasa, $2015(n=90)$ & $*$ & $*$ & $*$ & - & $* * * * * * * *$ & $*$ & $*$ & $*$ & 13 \\
\hline Calafiore, $2014(n=52)$ & $*$ & $*$ & $*$ & $* * * * * * *$ & $* * * *$ & $*$ & $*$ & $*$ & 16 \\
\hline Fattouch, $2012(\mathrm{n}=110)$ & - & $*$ & $*$ & $* * * * * *$ & $* * * * * * *$ & $*$ & $*$ & $*$ & 17 \\
\hline Langer, $2009(n=60)$ & $*$ & $*$ & $*$ & $* * * * *$ & $* * * * * * *$ & $*$ & $*$ & $*$ & 17 \\
\hline Borger, $2007(n=92)$ & - & $*$ & $*$ & $* * *$ & $* * * * * * * * * *$ & $*$ & $*$ & - & 17 \\
\hline
\end{tabular}

Quality scoring system based on modified Newcastle-Ottawa scale that includes pre-operative echocardiographic findings, clinical baseline and operative characteristics in terms ring size and of numbers of distal anastomoses.

Table 5. High sensitivity studies analysis $\infty$.

\begin{tabular}{|c|c|c|c|c|c|c|c|c|c|c|}
\hline \multirow[t]{2}{*}{ Outcome } & \multicolumn{3}{|l|}{$N=3$} & \multirow[t]{2}{*}{ Mean difference } & \multicolumn{3}{|c|}{ Overall effect } & \multicolumn{3}{|c|}{ Heterogeneity } \\
\hline & Studies & SVP & RA & & Odds ratio & $95 \% \mathrm{Cl}$ & $\mathrm{P}$ & $\mathrm{Chi}^{2}$ & $\mathrm{P}$ & $\mathrm{I}^{2}$ \\
\hline \multicolumn{11}{|l|}{ Primary outcome } \\
\hline Late recurrence of $M R^{a}$ & & 145 & 115 & & 0.39 & $0.16,0.95$ & 0.04 & 2.21 & 0.33 & $9 \%$ \\
\hline LVEDD & & 145 & 115 & 0.65 & & $-8.37,9.67$ & 0.89 & 37.63 & 0.00001 & $95 \%$ \\
\hline LVESD $^{a}$ & & 85 & 85 & -4.23 & & $-6.42,-2.04$ & 0.0002 & 0.14 & 0.71 & $0 \%$ \\
\hline$C D^{a}$ & & 115 & 85 & -3.99 & & $-4.15,-3.82$ & 0.0001 & 1.05 & 0.31 & $4 \%$ \\
\hline LVEF & & 115 & 85 & -4.79 & & $-13.73,3.78$ & 0.27 & 7.40 & 0.007 & $86 \%$ \\
\hline \multicolumn{11}{|l|}{ Secondary outcomes } \\
\hline CPB time & & 145 & 115 & 8.51 & & $-0.60,17.63$ & 0.07 & 4.16 & 0.12 & $52 \%$ \\
\hline$X C T^{a}$ & & 145 & 115 & 5.76 & & $0.62,10.90$ & 0.03 & 11.09 & 0.004 & $82 \%$ \\
\hline Early mortality & & 145 & 115 & & 0.93 & $0.35,2.44$ & 0.88 & 0.90 & 0.64 & $0 \%$ \\
\hline Mid-term survival & & 90 & 60 & & $0.76(\mathrm{HR})$ & $0.29,1.97$ & 0.56 & 1.88 & 0.17 & $46.8 \%$ \\
\hline
\end{tabular}

CD: coaptation depth; CPB: cardiopulmonary bypass; HR: hazard ratio; LVEDD: left ventricular end diastolic diameter; LVEF: left ventricular ejection fraction; LVESD: left ventricular end systolic diameter; MR: mitral regurgitation; RA: restrictive annuloplasty; SVP: sub-valvular procedures; XCT: cross-clamp time.

$\mathrm{a}=$ denote significance.

${ }^{\infty}$ High sensitivity studies include patients with coaptation depth $>1 \mathrm{~cm}$ or Tenting area $>2.5 \mathrm{~cm}^{2}$.

\section{Heterogeneity assessment: bias exploration}

In accordance with Cochrane guidelines, ${ }^{7}$ risk of bias analysis was performed for all studies included in this review (Figure 5). Overall, a high level of bias was detected, due to the non-randomized, unblinded nature of the majority of the studies. In addition to established bias assessment, a score was also given for each of the following: (1) multicenter trial, (2) propensity matched study and (3) confounder adjustment. No study fulfilled all three of these criteria (Figure 5). Two studies were propensity matched ${ }^{9,10}$ and one was corrected for poten- 


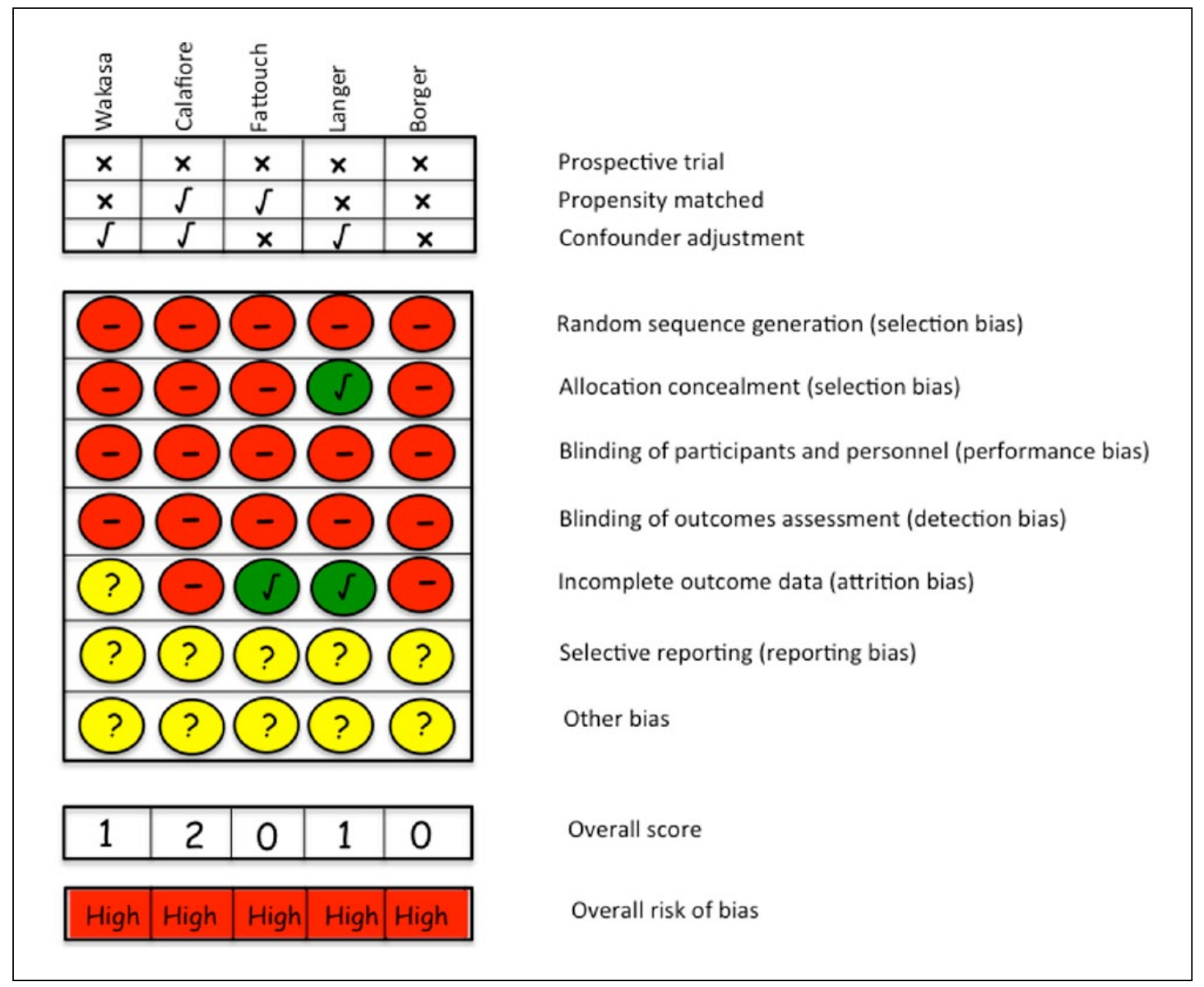

Figure 5. Risk of bias assessment.

tial echocardiographic confounders. ${ }^{11}$ Funnel plots were used to assess for publication bias for all primary and secondary outcomes. Very minor funnel plot asymmetry was identified for primary outcome (Figures $2 \mathrm{a}-\mathrm{b} /$ Figures $3 \mathrm{a}-\mathrm{b} /$ Figure 4).

\section{Comment}

This meta-analysis compares patients with coronary artery disease and moderate to severe cIMR undergoing either RA alone or annuloplasty with adjunctive SVP. Up to date, it is still unclear whether moderate cIMR has to be repaired at the moment of CABG since it may not be associated with clinically meaningful advantage or a higher degree of left ventricular reverse remodeling. ${ }^{3}$ There is a general agreement that severe cIMR has to be addressed, but the best surgical strategy, either repair or replacement, has yet to be established. ${ }^{10}$

Pathogenesis of cIMR is multifactorial and includes annular dilatation (Carpentier type I), restricted leaflet motion (Carpentier type IIIb) with different degrees of tethering attributable to papillary muscle displacement. ${ }^{11}$ In the context of moderate cIMR, the vast majorities of studies compared uniquely RA over CABG, with disputable results and many authors reported evidence of the recurrence of moderate to severe MR at follow-up. ${ }^{14}$ Taking into account the complex entity of the mitral valve in itself, continuous left ventricle remodeling and the diverse mechanisms underlying the etiology of cIMR, ${ }^{4} \mathrm{RA}$ alone may be not be sufficient to guarantee freedom from recurrence, reverse remodeling and clinical benefits. This is particularly true in the case of severe cIMR, where different reports have highlighted an expected more durable correction of mitral regurgitation in the replacement group over RA alone. ${ }^{3}$ To overcome this caveat, alternative repair techniques that directly tackle the subvalvular tethering forces, such as PMR, PMA and CC, have been proposed, with significantly better effects over RA alone. ${ }^{4}$

Interestingly, all the echocardiographic predictors for late repair failure, such as LVEDD $>65 \mathrm{~mm}$, posterior mitral leaflet angle $>45^{\circ}$, distal anterior mitral leaflet angle $>25^{\circ}$, systolic TA $>2.5 \mathrm{~cm}^{2}, \mathrm{CD}>10 \mathrm{~mm}$, end-systolic inter-papillary muscle distance $>20 \mathrm{~mm}$ and systolic sphericity index $>0.7$, have been derived from 
patients who underwent sole RA with no correction of the sub-valvular displacement forces. ${ }^{15}$

This is the first meta-analytic study to demonstrate that adjunctive sub-valvular mitral procedures to RA result in statistically significantly better freedom from MR recurrence, with reduced $C D$ and improvement of LVESD at mid-term follow-up (2.9 years) without increasing immediate surgical risk. Three studies specifically included patients with severe cIMR or the presence of predictors of repair failure as part of the sensitivity analysis. ${ }^{8,10,11}$ Borger $^{12}$ included patients with severe MR as well; however, data could not be separated from patients with moderate MR, hence, was not included in the sensitivity analysis. Calafiore ${ }^{9}$ excluded patients with $\mathrm{CD}>10 \mathrm{~mm}$ who directly underwent replacement with no attempt to repair. A classical restricted annuloplasty, as originally described by Bolling, ${ }^{1}$ was not used in the SVP groups, where a moderate downsizing or natural size technique was preferred. This possibly may be a favorable surgical philosophy in line with recent findings that show that RA might be associated with a higher trans-mitral gradient at rest and maximal exercise. However, correlation with functional capacity has yet to be determined. ${ }^{16}$ The adjunction of SVP led to statistically significantly prolonged $\mathrm{CPB}$ time and XCT. However, that did not increase the mortality rate (Table 3 ). In the surgical community, one of the most common reasons for crossover to CABG alone is the concern about the risk associated with valve repair. ${ }^{14}$ In a prospective randomized trial, Smith et al. observed that RA was associated with prolonged $\mathrm{CPB}$ and XCT time and a higher rate of stroke than CABG alone. ${ }^{14}$ In this meta-analysis, only Langer ${ }^{11}$ and Borger ${ }^{12}$ reported the incidence of major neurological events that was not statistically different between groups.

This study has limitations. Firstly, the limited number of studies included, although, despite the small number of patients involved, very limited heterogeneity for both primary and secondary outcomes was observed. Secondly, SVP included different techniques (PMR, PMA and CC) that may add intrinsic heterogeneity to the study. Nevertheless, all these procedures have the same rationale to act at the level of the structures below the mitral annulus with the aim to relieve mitral tenting or papillary muscle displacement.

With this study, we conclude that adding SVP to RA in a context of moderate to severe cIMR may be related with a reduced recurrence of late MR with preserved LVESD and reduced CD. However, prolonged CPB and $\mathrm{XCT}$ are required. These benefits are evident, even in patients with significant predictors of cIMR valve repair failure. Impact on late survival and functional class needs to be investigated.

\section{Declaration of Conflicting Interests}

The authors declared no potential conflicts of interest with respect to the research, authorship, and/or publication of this article.

\section{Funding}

The authors received no financial support for the research, authorship, and/or publication of this article.

\section{References}

1. Bolling SF, Deeb GM, Brunsting LA, Bach DS. Early outcome of mitral valve reconstruction in patients with endstage cardiomyopathy. J Thorac Cardiovas Surg 1995; 109: 676-682; discussion 682-673.

2. Levine RA, Schwammenthal E. Ischemic mitral regurgitation on the threshold of a solution: from paradoxes to unifying concepts. Circulation 2005; 112: 745-758.

3. Acker MA, Parides MK, Perrault LP, et al. Mitral-valve repair versus replacement for severe ischemic mitral regurgitation. New Engl J Med 2014; 370: 23-32.

4. Fattouch K, Castrovinci S, Murana G, Moscarelli M, Speziale G. Surgical management of moderate ischemic mitral valve regurgitation: where do we stand? World Journal of Cardiology 2014; 6: 1218-1222.

5. Moher D, Liberati A, Tetzlaff J, Altman DG; PRISMA Group. Preferred reporting items for systematic reviews and meta-analyses: the PRISMA statement. BMJ 2009; 339: b2535.

6. Stroup DF, Berlin JA, Morton SC, et al. Meta-analysis of observational studies in epidemiology: a proposal for reporting. Meta-analysis Of Observational Studies in Epidemiology (MOOSE) group. JAMA 2000; 283: 2008-2012.

7. The Cochrane Collaboration. Cochrane handbook for systematic reviews of interventions version 5.1.0 [updated March 2011] 2011.

8. Wakasa S, Shingu Y, Ooka T, Katoh H, Tachibana T, Matsui Y. Surgical strategy for ischemic mitral regurgitation adopting subvalvular and ventricular procedures. Ann Thorac Cardiovasc Surg 2015; 21: 370-377.

9. Calafiore AM, Refaie R, Iaco AL, et al. Chordal cutting in ischemic mitral regurgitation: a propensity-matched study. J Thorac Cardiovasc Surg 2014; 148: 41-46.

10. Fattouch K, Lancellotti P, Castrovinci S, et al. Papillary muscle relocation in conjunction with valve annuloplasty improve repair results in severe ischemic mitral regurgitation. J Thorac Cardiovasc Surg 2012; 143: 1352-1355.

11. Langer F, Kunihara T, Hell K, et al. Ring+string: successful repair technique for ischemic mitral regurgitation with severe leaflet tethering. Circulation 2009; 120: S85-91.

12. Borger MA, Murphy PM, Alam A, et al. Initial results of the chordal-cutting operation for ischemic mitral regurgitation. J Thorac Cardiovasc Surg 2007; 133: 1483-1492.

13. Manabe S, Shimokawa T, Fukui T, Tabata M, Takanashi S. Impact of papillary muscle approximation on mitral 
valve configuration in the surgical correction of ischemic mitral regurgitation. Thorac Cardiov Surg 2012; 60: 269-274.

14. Smith PK, Puskas JD, Ascheim DD, et al. Surgical treatment of moderate ischemic mitral regurgitation. New Engl J Med 2014; 371: 2178-2188.

15. Moscarelli M, Punjabi P. Predictors of recurrent chronic ischemic mitral regurgitation after mitral valve repair surgery. In: Fattouch KL, Lancellotti P, Angelini GD. eds. Secondary mitral valve regurgitation. Springer; 2015: 185-191.

16. Bertrand PB, Gutermann H, Smeets CJ, et al. Functional impact of transmitral gradients at rest and during exercise after restrictive annuloplasty for ischemic mitral regurgitation. J Thorac Cardiovasc Surg 2014; 148: 183-187. 